Research Paper

\title{
Effects of perioperative Eicosapentaenoic acid-enriched oral nutritional supplement on lean body mass after total gastrectomy for gastric cancer
}

\author{
Toru Aoyama $1^{*}$, Takaki Yoshikawa $1^{* \bowtie}$, Satoshi Ida 2, Haruhiko Cho 1, Kentaro Sakamaki 34, Yuichi Ito 5 , \\ Kazumasa Fujitani ${ }^{6}$, Nobuhiro Takiguchi ${ }^{7}$, Yoshiyuki Kawashima ${ }^{8}$, Kazuhiro Nishikawa 9, Takashi \\ Oshima ${ }^{1}$, Souya Nunobe ${ }^{2}$, Naoki Hiki ${ }^{10}$ \\ 1. Kanagawa Cancer Center, Department of Gastrointestinal Surgery, Yokohama, Japan. \\ 2. Department of Gastroenterological Surgery, Cancer Institute Hospital of Japanese Foundation for Cancer Research, Tokyo, Japan \\ 3. Department of Biostatistics and Bioinformatics, Graduate School of Medicine, The University of Tokyo, Tokyo, Japan \\ 4. Department of Biostatistics, School of Medicine, Yokohama City University, Yokohama, Japan \\ 5. Aichi Cancer Center Hospital, Department of Gastroenterological Surgery, Nagoya, Japan \\ 6. Osaka General Medical Center, Department of Surgery, Osaka, Japan \\ 7. Division of Gastrointestinal Surgery, Chiba Cancer Center, Chiba, Japan \\ 8. Saitama Cancer Center, Saitama, Japan \\ 9. Department of Surgery, National Hospital Organization Osaka National Hospita, Osaka, Japan \\ 10. Department of Upper Gastrointestinal Surgery Kitasato University School of Medicine, Sagamihara, Japan \\ *These two authors contributed equally.
}

$\square$ Corresponding author: Takaki Yoshikawa, MD, PhD, Department of Gastric Surgery, National Cancer Center, 5-1-1 Tsukiji, Chuo-ku, Tokyo, Japan, E-mail: tayoshik@ncc.go.jp, Telephone: +81-3-3542-2511

(c) Ivyspring International Publisher. This is an open access article distributed under the terms of the Creative Commons Attribution (CC BY-NC) license (https://creativecommons.org/licenses/by-nc/4.0/). See http://ivyspring.com/terms for full terms and conditions.

Received: 2018.08.31; Accepted: 2019.01.04; Published: 2019.01.29

\begin{abstract}
Background: In previous our phase III study to compare perioperative standard diet with or without Eicosapentaenoic acid (EPA)-enriched oral nutritional supplement (EPA-ON), additional EPA-ON did not contribute to prevent body weight loss after total gastrectomy. This report clarified whether EPA-ON could prevent loss of lean body mass (LBM) after total gastrectomy, a key secondary endpoint, in our phase III trial.

Methods: This phase III study was designed as multicenter, open-label, superiority, randomized trial to confirm the preventive effect of EPA-ON body weight loss after total gastrectomy for gastric cancer. Eligible patients were randomized to either Standard-diet group or EPA-ON group by a centralized dynamic method. Standard-diet group was given no additional nutritional supplementation perioperatively (standard diet), while EPA-ON group was given an EPA-enriched supplement (ProSure ${ }^{\circledR}$, Abbott Japan, Tokyo, Japan) in addition to their standard diet. This supplement included $600 \mathrm{kcal}$ with $2.2 \mathrm{~g} /$ day of EPA. For both groups, patients underwent total gastrectomy with Roux-en $Y$ reconstruction.

Results: A total of 123 patients (Group A: 60, Group B: 63) were analyzed in the study. All background factors were well balanced between the both groups. Median loss of LBM was $6.74 \%$ (range $-3.91 \%$ to $20.27 \%$ ) in the Standard-diet group and $6.89 \%$ (range $-5.11 \%$ to $20.04 \%$ ) in the EPA-ON group at 1 month after surgery and was $8.59 \%$ (range $-4.40 \%$ to $20.27 \%$ ) in the Standard-diet group and $7.77 \%$ (range $-5.57 \%$ to $23.35 \%$ ) in the EPA-ON group at 3 months after surgery, which was not significantly different at the both $(\mathrm{p}=0.794$ and $\mathrm{P}=0.393$, respectively).

Conclusions: The perioperative EPA-ON could not be recommended to prevent loss of LBM after total gastrectomy.
\end{abstract}

Key words: gastric cancer, EPA, gastrectomy, lean body mass loss

\section{Introduction}

Gastric cancer had 951,600 new cancer cases and 723,100 deaths occurred in 2012 [1]. When gastric

cancer is limited to the local site, surgical resection is mainstay for the cure of gastric cancer. However, 
gastrectomy has a risk of surgical invasion and loss of the stomach. Among post-gastrectomy symptoms, body weight loss is unavoidable after gastrectomy 2,3. Previous studies demonstrated that weight loss decreased nutritional status, postoperative quality of life, compliance of S-1 adjuvant chemotherapy which could lead to the poor survival $[4,5]$. Weight loss after total gastrectomy may occur through various mechanisms, such as hyper catabolism associated with inflammatory reactions due to surgical stress, reduced food intake owing to loss of reservoir function, and reduction in blood ghrelin level [6].

Eicosapentaenoic acid (EPA), a long-chain polyunsaturated fatty acid of the omega-3 (n-3) family, has both anabolic and immunomodulatory properties, making it attractive for use during the postoperative period. Ryan et al reported that enteral nutrition including EPA tended to preserve the body weight and lean body mass after esophagectomy in a double blinded randomized study, suggesting that EPA has benefits even for patients who undergo major surgery [7]. Based on these, we conducted the prospective randomized phase III trial to evaluate whether the perioperative administration of an EPA-enriched oral nutritional supplement (EPA-ON) can prevent body weight loss after total gastrectomy for gastric cancer [8]. However, additional EPA-ON did not contribute to prevent weight loss in our phase III study. Body weight consists of fat mass and lean body mass (LBM). Because loss of fat mass is a major contributor for body weight loss observed after gastrectomy [9], effects of EPA-ON on LBM could be masked behind body weight lose including loss of fat mass. Recently, we reported that decrease of $5 \%$ or more in LBM increased toxicity and decreased compliance of adjuvant chemotherapy with S-1 in stage II /III gastric cancer patients. Thus, it must be clinically interesting to see whether EPA-ON could prevent only $5 \%$ decrease in LBM [10].

LBM is a key secondary endpoint of our phase III study. This report clarifies whether additional EPA-ON prevents loss of LBM after total gastrectomy in this phase III study.

\section{Patients and Methods}

The present study was designed as multicentre, open-label, superiority, randomized phase III trial to evaluate the preventive effect of EPA on body weight loss after total gastrectomy for gastric cancer. This clinical trial was registered at the University Hospital Medical Information Network Clinical Trials Registry (UMIN000006380). Patients were recruited between October 2011 and July 2014. The details of this trial were described in the previous report $[8,11]$. Briefly, key eligibility included histologically proven adenocarcinoma of the stomach, clinical T1-T4a and M0, the possibility of R0 resection by total gastrectomy, sufficient oral intake and organ function, and age ranging between 20 and 80 years. Eligible patients were randomized to either Standard-diet group or EPA-ON group by a centralized dynamic method. Standard-diet group was given no additional nutritional supplementation perioperatively (standard diet), while EPA-ON group was given an EPA-enriched supplement (ProSure ${ }^{\circledR}$, Abbott Japan, Tokyo, Japan) in addition to their standard diet. This supplement included $600 \mathrm{kcal}$ with $2.2 \mathrm{~g} /$ day of EPA. The hospital diet was not restricted. The amount of the supplements consumed was counted. The supplement was given from 7 days to 1 day before surgery. For patients undergoing total gastrectomy, regardless of whether it was curative resection, the supplement was given for 21 days when oral intake was initiated after total gastrectomy. Perioperative care other than EPA-enriched supplement followed routine clinical practice performed in each institution in both groups. In the present study, we decided that $30 \%$ reduction in \% body weight loss is necessary for this test treatment, considering the balance between the risk and benefit. From the retrospective data in our institution, we estimated \% body weight loss as $8.5 \%$ at 1 month and $11.0 \%$ at 3 months in the control arm. Considering 30\% risk reduction, $\%$ body weight loss was estimated as $6.0 \%$ at 1 month and $7.7 \%$ at 3 months in the test arm. Thus, we assumed that the expected difference in the \% body weight loss between both arms would be $2.5 \%$ (SD $4.0 \%$ ) and 3.3\% (SD 5.5\%) at 1 and 3 months, respectively. In this situation, the sample size required to ensure an at least $80 \%$ probability for both hypotheses was 110 patients, with 55 patients per arm. Considering the likelihood of enrolling ineligible patients, the number of patients to be accrued was set at 120 in total.

\section{Surgery}

For both groups, the patients underwent open or laparoscopic total gastrectomy with Roux-en $\mathrm{Y}$ reconstruction. The extent of dissection principally followed the third edition of the Gastric Cancer Treatment Guideline published by the Japanese Gastric Cancer Association [12, 13]. Spleen-preserving D2 total gastrectomy was permitted in this study.

\section{Perioperative data collection}

The body composition was assessed using multifrequency bioelectrical impedance with eight tactile electrodes (MC-190EM; Tanita, Tokyo, Japan, or InBody 720; Biospace, Tokyo, Japan). Various parameters, including body weight, body mass index (BMI), and lean body mass (LBM) were automatically and simultaneously measured. The body composition 
was measured before the study entry and 1 and 3 months after surgery.

\section{Evaluation and statistical methods}

Progression of tumors was evaluated by the $14^{\text {th }}$ edition of the Japanese Gastric Cancer Classification [13]. The relative performance (\%) of the oral nutritional supplement was calculated by "actual dose $\times 100$ / planned dose. Lean body mass (LBM) loss at 1 month was defined as \% LBM loss = (preoperative LBM - LBM at 1 month after surgery) $\times$ 100 / preoperative LBM. We also defined LBM loss at 3 month by the same way. Binary endpoints of LBM loss at 1 and 3 month were defines as severe group when LBM loss was of $5 \%$ or more and as mild group when LBM loss was less than $5 \%$.

The continuous variables were expressed as the median with range or mean \pm standard deviation (SD), the categorical variables were express by frequencies. For the LBM losses, the means and their 95\% confidence intervals was estimated and compared with Student's $t$-test. The binary endpoints of LBM loss were compared using the Fisher's exact test. The interaction between subgroups was examined by Breslow-Day test. The statistical analyses were performed using the SAS software program, version 9.3 (SAS Institute, Cary, NC, USA). A two-sided significance level of $P<0.05$ was used for all of the statistical analyses.

\section{Results}

\section{Patient's Characteristics}

CONSORT diagram is shown in Figure 1. Between October 2011 and July 2014, 127 patients were enrolled in this study. Because 1 patient was rejected to receive the protocol treatment after the registration, 126 patients were randomized to Standard-diet group (63 patients) or EPA-ON group (63 patients). Of the 63 patients in the Standard-diet group, 2 were excluded from the safety analysis due to unresectable tumors for peritoneal dissemination and an additional 1 patient was excluded from the efficacy analysis due to missing data of body weight at the enrollment. One-hundred twenty-three patients baseline characteristics and operative details are shown are summarized in Table 1 . The background factors and operative procedures were well balanced between the two arms. Median relative performance of supplement in EPA-ON group was $100 \%$ before surgery and $54 \%$ after surgery.

\section{Operative Morbidity and Mortality}

Postoperative morbidities were observed in 8 patients of Standard-diet group $(13.1 \%)$ and in 9 patients $(14.3 \%)$ of EPA-ON group (14.3\%). Overall, pancreatic fistula, abdominal abscess, and anastomotic leakage developed in $3.2 \%, 2.4 \%$, and $0.8 \%$ of patients, respectively. There was no relationship between the administration of EPA and the occurrence of postoperative complications.

\section{Loss of LBM after surgery}

Median loss of LBM was $6.74 \%$ (range $-3.91 \%$ to $20.27 \%$ ) in the Standard-diet group and $6.89 \%$ (range $-5.11 \%$ to $20.04 \%$ ) in the EPA-ON group at 1 month after surgery and was $8.59 \%$ (range $-4.40 \%$ to $20.27 \%$ ) in the Standard-diet group and $7.77 \%$ (range $-5.57 \%$ to $23.35 \%$ ) in the EPA-ON group at 3 months after surgery, which was not significantly different at the both ( $p=0.794$ and $p=0.393$, respectively) (Table 2).

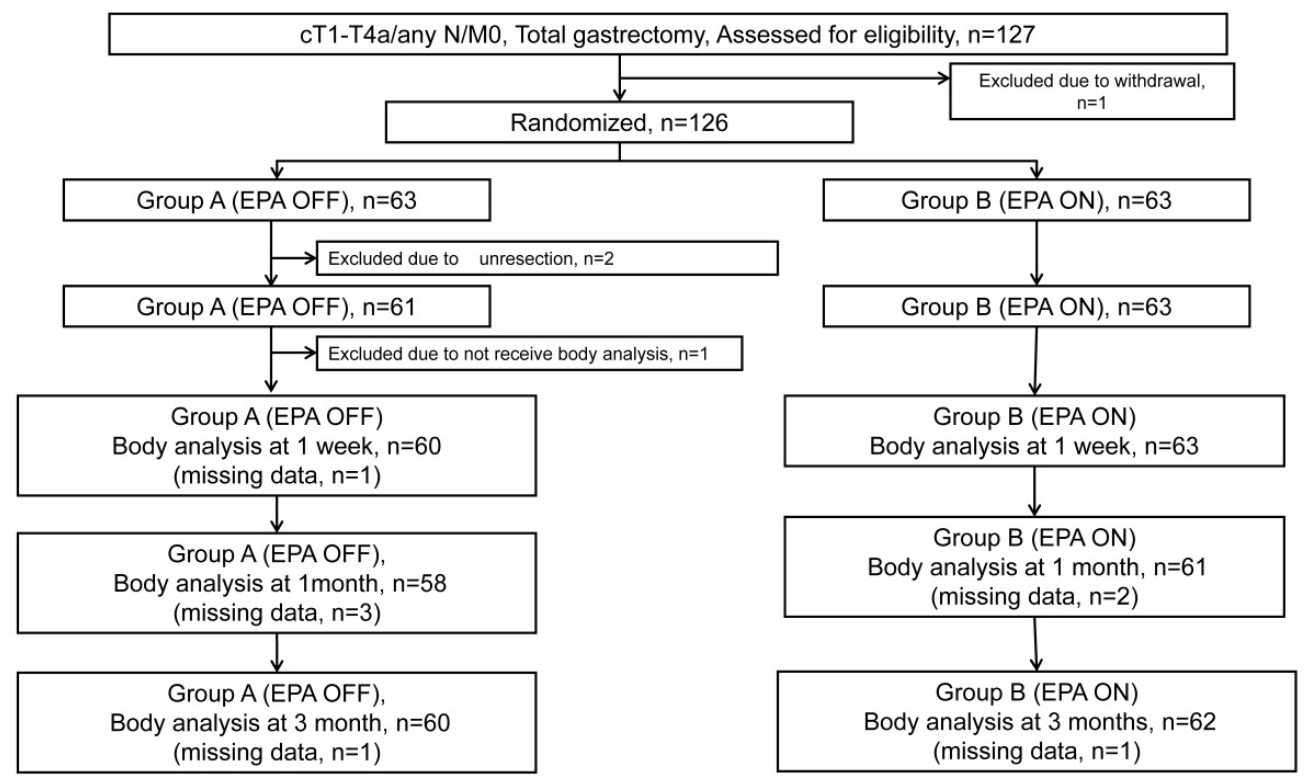

Figure 1. Flow diagram of 127 patients 
Table 1. Background characteristics between standard diet group and EPA-enriched supplemental group

\begin{tabular}{|c|c|c|}
\hline & $\begin{array}{l}\text { Standard diet } \\
\text { group }(n=60)\end{array}$ & $\begin{array}{l}\text { EPA-enriched } \\
\text { supplemental group } \\
(\mathrm{n}=63)\end{array}$ \\
\hline Median age (years) (range) & $65.6(30-80)$ & $65.1(31-79)$ \\
\hline Gender (Male/Female) & $43 / 17$ & $46 / 17$ \\
\hline Preoperative mean body weight $(\mathrm{kg})$ & $47.8 \pm 8.6$ & $47.1 \pm 9.8$ \\
\hline Mena height $(\mathrm{cm})$ & $163.7 \pm 8.0$ & $160.6 \pm 8.4$ \\
\hline Preoperative mean lean body mass $(\mathrm{kg})$ & $47.0 \pm 7.5$ & $45.7 \pm 9.3$ \\
\hline Preoperative serum albumin $(\mathrm{mg} / \mathrm{dl})$ & $4.2 \pm 0.4$ & $4.1 \pm 0.5$ \\
\hline Preoperative C-reactive protein $(\mathrm{mg} / \mathrm{dl})$ & $0.2 \pm 0.5$ & $0.3 \pm 0.5$ \\
\hline $\begin{array}{l}\text { Location of primary tumor (Upper } \\
\text { third/Middle third/Lower third) }\end{array}$ & $35 / 24 / 0$ & $42 / 17 / 4$ \\
\hline Clinical T factor (T1/T2/T3/T4) & $16 / 10 / 11 / 23$ & $12 / 13 / 12 / 26$ \\
\hline Clinical N factor (negative/ positive) & $39 / 21$ & $40 / 23$ \\
\hline $\begin{array}{l}\text { Surgical approach } \\
\text { (conventional/laparoscopic) }\end{array}$ & $47 / 13$ & $52 / 11$ \\
\hline $\begin{array}{l}\text { Extent of lymph node dissection } \\
\text { (D0/D1/D2/D3) }\end{array}$ & $1 / 15 / 43 / 1$ & $0 / 10 / 53 / 0$ \\
\hline Operation time (min) (range) & $295(83-523)$ & $296(145-510)$ \\
\hline Blood loss (ml) (range) & $320(0-2080)$ & $340(0-3560)$ \\
\hline \multicolumn{3}{|l|}{ Postoperative morbidity } \\
\hline Overall & 8 & 9 \\
\hline Pancreatic fistula & 2 & 2 \\
\hline Abdominal abscess & 1 & 2 \\
\hline Anatomic leakage & 1 & 0 \\
\hline Bleeding & 0 & 1 \\
\hline Others & 4 & 4 \\
\hline
\end{tabular}

Table 2. Comparison change of lean body mass between standard diet group and EPA-enriched supplemental group

\begin{tabular}{llll}
\hline & $\begin{array}{l}\text { Standard diet group } \\
(\mathrm{n}=60)\end{array}$ & $\begin{array}{l}\text { EPA-enriched supplemental } \\
\text { group }(\mathrm{n}=63)\end{array}$ & $\begin{array}{l}\mathrm{P} \\
\text { value }\end{array}$ \\
\hline $\begin{array}{l}1 \text { month after } \\
\text { surgery }\end{array}$ & $-6.74 \%(-20.27$ to 3.91$)$ & $-6.89 \%(-20.04$ to 5.11$)$ & 0.794 \\
$\begin{array}{l}3 \text { month after } \\
\text { surgery }\end{array}$ & $-8.59 \%(-20.27$ to 4.40$)$ & $-7.77 \%(-23.35$ to 5.57$)$ & 0.393 \\
\hline
\end{tabular}

Figure 2 showed the waterfall plot showing each LBM loss put from the smaller value to the higher one in each group. Severe LBM loss of $5 \%$ or more at 1 month after surgery was observed in 44 patients $(80.0 \%)$ in the Standard-diet group and in 37 patients $(67.3 \%)$ in the EPA-ON group ( $\mathrm{p}=0.194$, Figure 2$)$, while that at 3 months after surgery was found in 51 patients $(91.1 \%)$ in the Standard-diet group and in 43 patients $(76.8 \%)$ in the EPA-ON group $(p=0.070$, Figure 3). EPA-ON tended to prevent meaningful loss of LBM after total gastrectomy (Table 3).

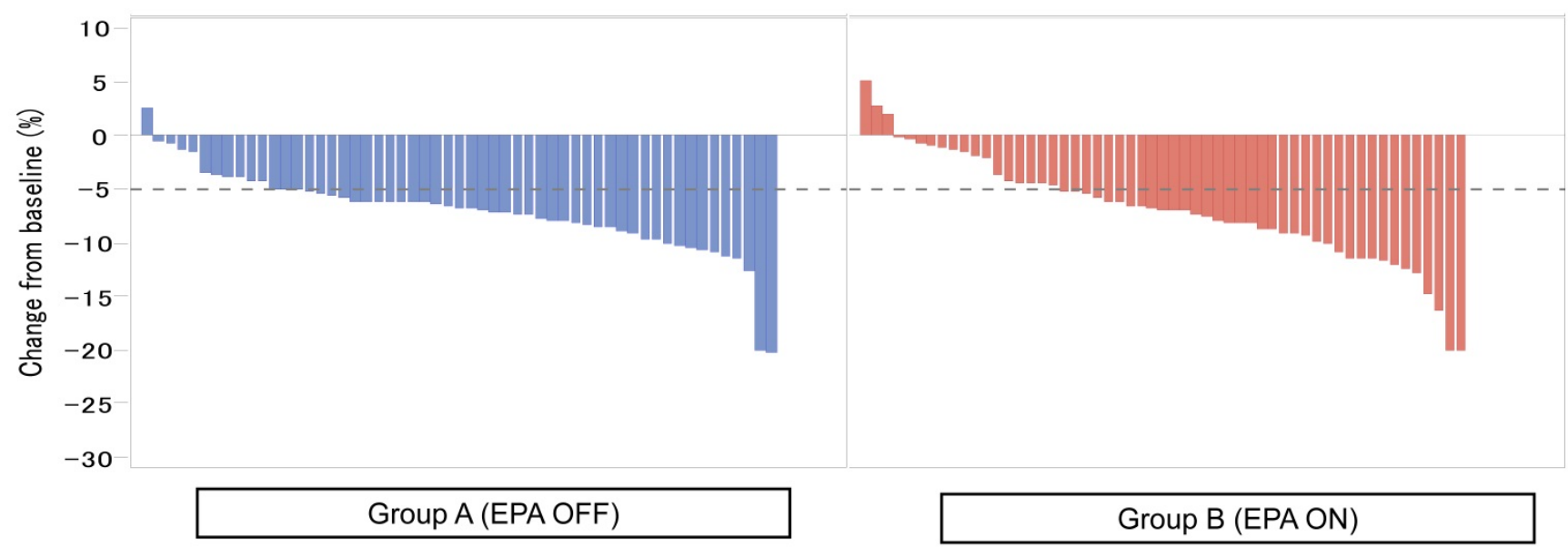

Figure 2. Percent of loss of lean body mass at 1 month between EPA-enriched supplement arm and standard diet arm.

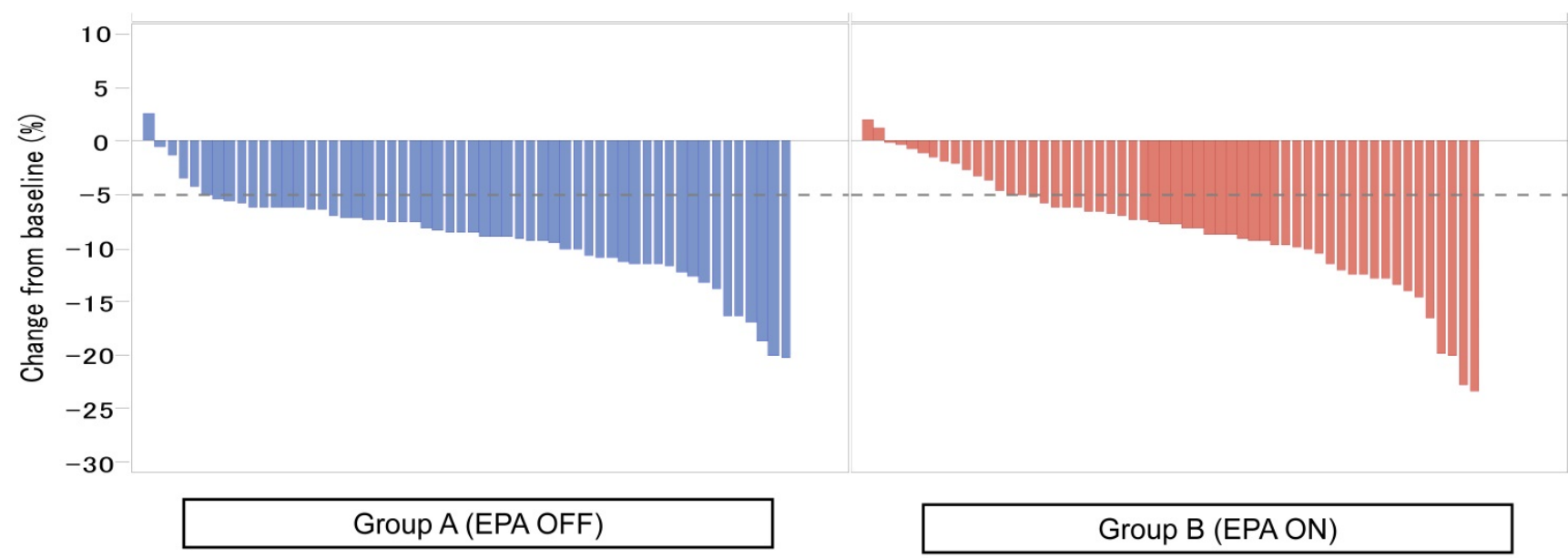

Figure 3. Percent of loss of lean body mass at 3 months between EPA-enriched supplement arm and standard diet arm. 


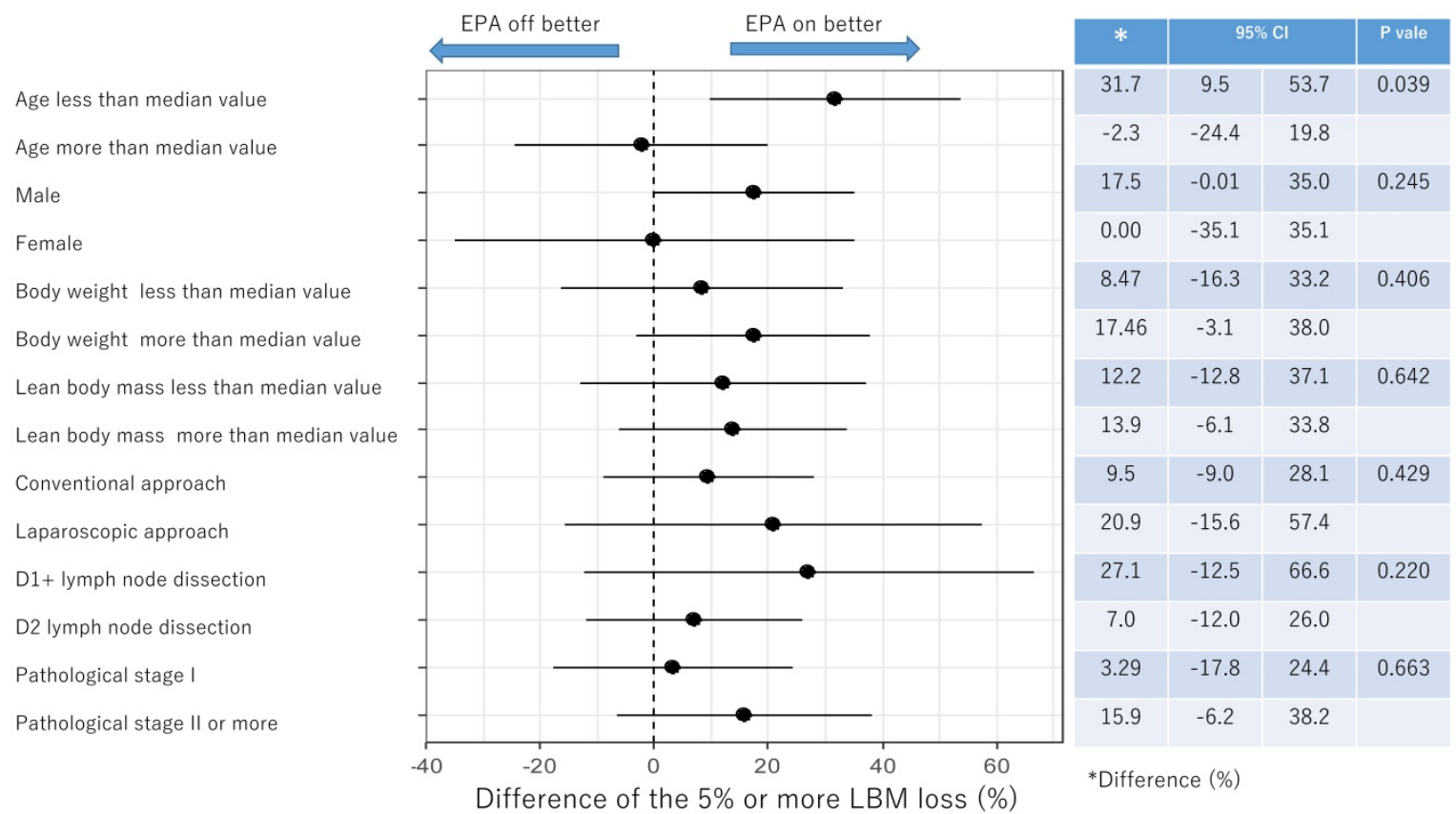

Figure 4. Subgroup analysis of $5 \%$ or more LBM loss at 1 month after surgery

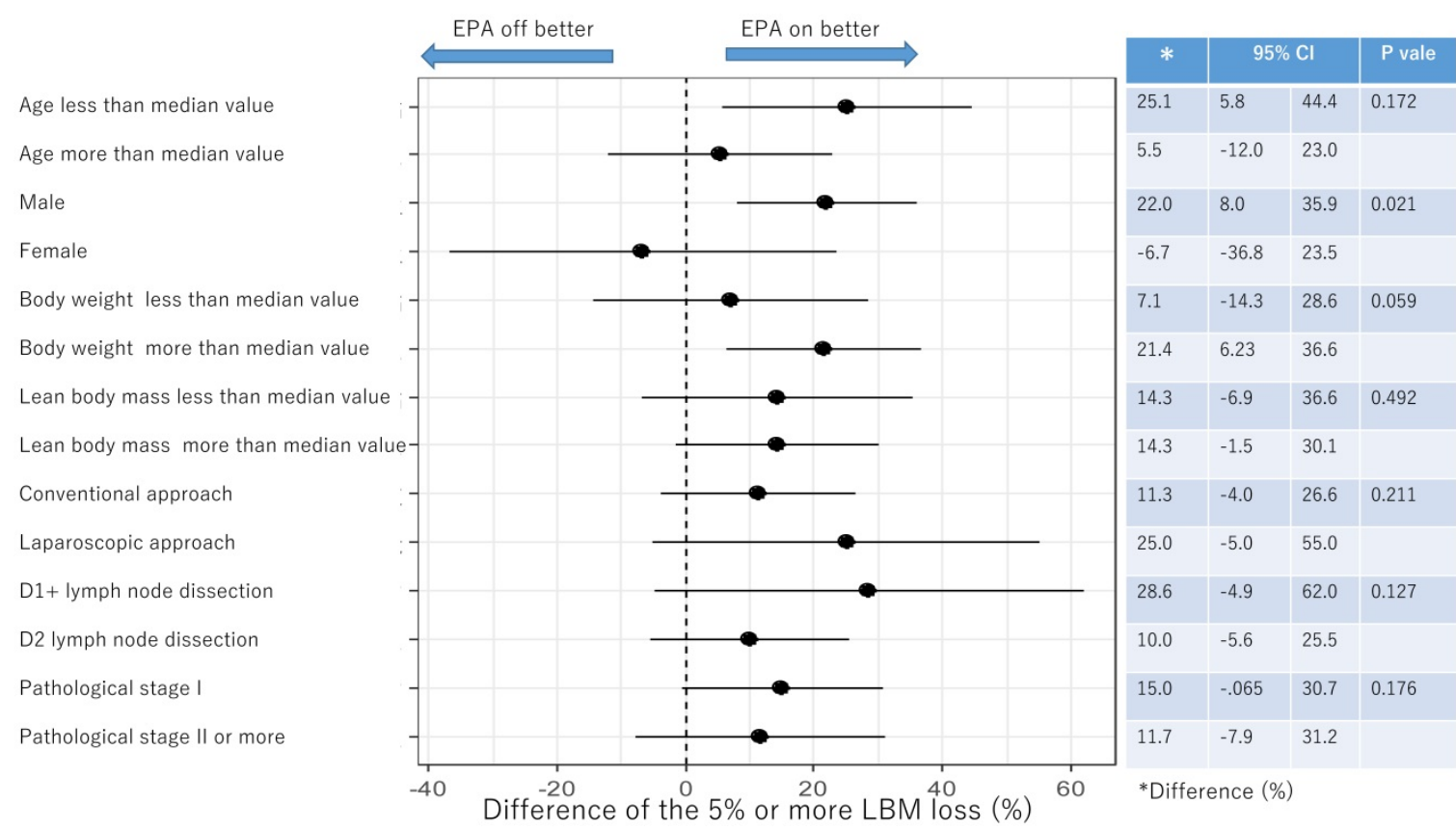

Figure 5. Subgroup analysis of $5 \%$ or more LBM loss at 3 months after surgery

Table 3. Comparison change of lean body mass $\geq 5 \%$ between standard diet group and EPA-enriched supplemental group

\begin{tabular}{llll}
\hline & $\begin{array}{l}\text { Standard diet } \\
\text { group }(\mathrm{n}=60)\end{array}$ & $\begin{array}{l}\text { EPA-enriched } \\
\text { supplemental group } \\
(\mathrm{n}=63)\end{array}$ & P value \\
\hline 1 month after surgery & 44 patients $(80 \%)$ & 37 patients $(67.3 \%)$ & 0.194 \\
3 month after surgery & 51 patients $(91.1 \%)$ & 43 patients $(76.8 \%)$ & 0.070 \\
\hline
\end{tabular}

\section{Subgroup analysis for proportion of $5 \%$ or more LBM loss after surgery}

Figure 4 and Figure 5 showed the difference in the proportion of binary endpoints between
Standard-diet group and EPA-suppl group. The significant and marginal differences between subgroups were observed in the age and gender.

\section{Discussion}

Although EPA-enriched immunonutrition had no preventive effects on loss of body weight after total gastrectomy, its effect on the loss of LBM had remained unclear. The major finding of the present study was that EPA-ON had no significant effects on not only loss of absolute value of LBM but also clinically meaningful loss of LBM separated by two 
categories. Thus, the perioperative EPA-ON could not be recommended to prevent loss of LBM after total gastrectomy.

Very recently, Healy et al just reported that body composition and nutritional status during 6 months after esophagectomy were not different by adding EPA to enteral nutrition (EN) in a phase III study [14]. Thus, two large phase III studies failed to demonstrate preventing effect of LBM loss after upper gastrointestinal surgery. Different from the present study in which compliance after surgery was low, $54 \%$, their study achieved high overall compliance rate of $96 \%$. Thus, negative results in the present study would not be explained only by poor compliance of EPA.

Why did EPA fail to show efficacy? Loss of LBM after surgery is due mainly to the increased inflammatory activity. Theoretically, EPA has both anabolic and immunomodulatory properties for surgical stress [15]. EPA may modulate this both directly through eicosanoid production and indirectly through decreased production of TNF, IL-6, and related cytokines [16]. Actually, previous prospective randomized controlled trial tested whether perioperative infusions of omega- 3 modify the cell membrane composition, inflammatory responses, and clinical course of patients undergoing elective coronary artery bypass surgery [17]. That trial showed that omega-3 significantly decreased the IL-6 response and trended to decrease the IL- 8 response on day 1 after surgery. In addition, there is also evidence for a muscle anabolic effect of EPA. EPA supplementation had been reported to help maintain whole-body protein synthesis, whole-body protein net balance, and muscle mass in burned rats and tumor-bearing mice [18]. On the other hands, decreased calorie intake and/or decreased physical activity which were observed after gastrointestinal surgery also affect the LBM loss [19]. These factors, such as decreased calorie intake and/or decreased physical activity might offset the effect of the anti-inflammatory properties of EPA.

The subgroup analyses demonstrated that efficacy of EPA-ON to prevent severe clinically meaningful loss of LBM was different depending on age or gender although we could not deny possibility of statistical error by multiple analyses. First, we discuss on the relation between age and loss of LBM. Efficacy of EPA-ON was observed in the non-elderly patients but not in the elderly patients. Muscle anabolic response is known to be attenuated with aging [20]. Previous reports showed that muscle protein synthesis rates were $20 \%$ lower in the older adults as compared to the young adults [21]. Therefore, even though EPA showed a similar anti-inflammatory and anabolic effect for LBM after surgery, the elderly might have negated the anti-inflammatory and anabolic effect of EPA due to aging. Second, we discuss about the relation between gender and loss of LBM. Several previous studies showed that effects of EPA on muscle catabolism were different depending on gender [22]. However, these mechanisms proposed for the subgroup analysis were currently speculative.

In the present study, there were some limitations. First, the segmental body composition was analyzed using a bioelectrical impedance analyzer, which could not directly measure the muscle mass. The lean body mass is calculated from the sum of the muscle mass and the mass of the visceral organs. However, the mass of visceral organs would not be changed by surgery, thus the major contributor to the change in the lean body mass would be a change in the muscle mass. Moreover, comparability between the both groups would be preserved even though the measurement included some errors. Second, the body composition was not measured at the same time in one day. In the present study, the body composition was measured at the morning before the breakfast before and 1 week after surgery, while the time for the measurement was not pre-specified at 1 and 3 months after surgery. In addition, the perioperative care was not standardized between the institutions. These were also one of limitations of the present study because body water is influenced by oral intake.

In conclusions, the present study demonstrated that EPA-enriched oral nutritional supplement had no significant preventive effects on not only loss of absolute value of LBM but also clinically meaningful loss of LBM separated by two categories. The perioperative EPA could not be recommended to prevent loss of body weight or LBM after total gastrectomy.

\section{Acknowledgements}

This work was supported by non-governmental organization, the Kanagawa Standard Anti-Cancer Therapy Support System (KSATSS). The authors express their sincere gratitude to Ms. Natsumi Sato and Ms. Rika Takahashi for their excellent data management in this study.

\section{Human Rights Statement and Informed Consent}

The study data and informed consent were obtained in accordance with the Declaration of Helsinki and were approved by the Ethics Review Board of Kanagawa Cancer Center. Informed consent or substitute for it was obtained from all patients for being included in the study. 


\section{Competing Interests}

The authors have declared that no competing interest exists.

\section{References}

1. Torre LA, Bray F, Siegel RL, et al. Global cancer statistics, 2012. CA Cancer J Clin. 2015; 65: 87-108.

2. Adams JF. The clinical and metabolic consequences of total gastrectomy. I. Morbidity, weight, and nutrition. Scand J Gastroenterol 1967; 2: 137-49.

3. Fein M, Fuchs KH, Thalheimer A, et al. Long-term benefits of Roux-en-Y pouch reconstruction after total gastrectomy: a randomized trial. Ann Surg 2008; 247: 759-765.

4. Aoyama T, Yoshikawa T, Shirai J, et al. Body weight loss after surgery is an independent risk factor for continuation of S-1 adjuvant chemotherapy for gastric cancer. Ann Surg Oncol. 2013; 20: 2000-6.

5. Aoyama T, Sato T, Maezawa Y, et al. Postoperative weight loss leads to poor survival through poor S-1 efficacy in patients with stage II/III gastric cancer. Int J Clin Oncol. 2017; 22: 476-483.

6. Ryan AM, Healy LA, Power DG, et al. Short-term nutritional implications of total gastrectomy for malignancy, and the impact of parenteral nutritional support. Clin Nutr.2007; 26: 718-727.

7. Ryan AM, Reynolds JV, Healy L, et al. Enteral nutrition enriched with eicosapentaenoic acid (EPA) preserves lean body mass following esophageal cancer surgery: results of a double-blinded randomized controlled trial. Ann Surg 2009;249:355-63.

8. Ida S, Hiki N, Cho H, et al. Randomized clinical trial comparing standard diet with perioperative oral immunonutrition in total gastrectomy for gastric cancer. Br J Surg. 2017; 104: 377-383.

9. Yamaoka $Y$, Fujitani $\mathrm{K}$, Tsujinaka $\mathrm{T}$, et al. Skeletal muscle loss after total gastrectomy, exacerbated by adjuvant chemotherapy. Gastric Cancer. 2015; 18: 382-9.

10. Aoyama T, Kawabe T, Fujikawa H, et al. Loss of Lean Body Mass as an Independent Risk Factor for Continuation of S-1 Adjuvant Chemotherapy for Gastric Cancer. Ann Surg Oncol. 2015; 22: 2560-6.

11. Yoshikawa T, Hiki N, Taguri M, et al. A Phase III trial to evaluate the effect of perioperative nutrition enriched with eicosapentaenoic acid on body weight loss after total gastrectomy for T2-T4a gastric cancer. Jpn J Clin Oncol. 2012; 42: $459-62$.

12. Japanese Gastric Cancer Association. Japanese gastric cancer treatment guidelines 2010 (ver. 3). Gastric Cancer. 2011; 14: 113-23.

13. Japanese Gastric Cancer Association. Japanese classification of gastric carcinoma: 3rd English edition. Gastric Cancer. 2011; 14: 101-12.

14. Healy LA, Ryan A, Doyle SL, et al. Does Prolonged Enteral Feeding With Supplemental Omega-3 Fatty Acids Impact on Recovery Post-esophagectomy: Results of a Randomized Double-Blind Trial. Ann Surg. 2017; 266: 720-728.

15. Fritsche K. Fatty acids as modulators of the immune response. Annu Rev Nutr. 2006; 26: 45-73.

16. Barber MD, Fearon KC, Tisdale MJ, et al. Effect of a fish oil-enriched nutritional supplement on metabolic mediators in patients with pancreatic cancer cachexia. Nutr Cancer. 2001; 40: 118-124.

17. Berger MM, Delodder F, Liaudet L, et al. Three short perioperative infusions of n-3 PUFAs reduce systemic inflammation induced by cardiopulmonary bypass surgery: a randomized controlled trial. Am J Clin Nutr. 2013; 97: 246-54.

18. Smith GI, Atherton P, Reeds DN, et al. Omega-3 polyunsaturated fatty acids augment the muscle protein anabolic response to hyperinsulinaemia-hyperaminoacidaemia in healthy young and middle-aged men and women. Clin Sci (Lond). 2011; 121: 267-78.

19. Noguchi $Y$, Tsuburaya A, Makino T, et al. Metabolic alteration in totally gastrectomised patients-caloric intake and energy con-sumption. Asian J Surg. 1992; 15: 97-102.

20. Lalia AZ, Dasari S, Robinson MM, et al. Influence of omega-3 fatty acids on skeletal muscle protein metabolism and mitochondrial bioenergetics in older adults. Aging (Albany NY). 2017; 9: 1096-1129.

21. Toth MJ, Matthews DE, Tracy RP, et al. Age-related differences in skeletal muscle protein synthesis: relation to markers of immune activation. Am J Physiol Endocrinol Metab. 2005; 288: E883-91.

22. Da Boit M, Sibson R, Sivasubramaniam S, et al. Sex differences in the effect of fish-oil supplementation on the adaptive response to resistance exercise training in older people: a randomized controlled trial. Am J Clin Nutr. 2017; 105: 151-158. 